\title{
Choroid Plexus Cyst in the Lateral Ventricle Causing Obstructive Symptoms in an Adult
}

Robert J. Dempsey, M.D., and William F. Chandler, M.D.

This case of a rare symptomatic choroid plexus cyst in the lateral ventricle, which presented with papilledema and decreased vision, is the first such lesion found in an adult. Five earlier reported cases, all in children, are discussed, as are the limitations of computerized tomographic (CT) scanning and the need for further radiological studies to establish the diagnosis preoperatively. Metrizamide ventriculography with CT scanning is used to define the lesion and plan an operative approach.

Small cysts of the choroid plexus in the lateral ventricle are a relatively common finding at autopsy. Netsky and Shuangshoti [10] noted choroid plexus tubules in folded neuroepithelium in some $30 \%$ of cases studied. These are generally less than $1 \mathrm{~cm}$ in diameter and do not cause obstructive symptoms. To our knowledge, only 5 cases of symptomatic cysts of the choroid plexus of the lateral ventricle have been described in the literature $[1,2,6,7$, 9]. An additional clinical case was reported by Brown in 1876 but was never confirmed histologically [4]. All of these cases were in children under the age of 10 , and only 1 was discovered since the advent of computerized tomographic $(\mathrm{CT})$ scanning. The present case is the first report of a symptomatic lateral ventricular choroid plexus cyst in an adult; the combination of CT scanning and ventriculography, both with intraventricular metrizamide, was used to diagnose this rare condition and plan operative intervention.

\section{Case Report}

The patient was a 21 -year-old right-handed woman test driver who presented with a one-month history of gradual blurring of vision to the point of making reading impossible. She also complained of episodic headaches during this period; the headaches were moderately severe and associated with nausea, but there was no vomiting, neck stiffness, focal weakness, or numbness. During the week prior to ad-

From the Department of Surgery, Section of Neurosurgery, University of Michigan Hospital, Ann Arbor, MI 48109.

Address reprint requests to Dr. William F. Chandler.

Key words: lateral ventricle; neoplasm; choroid plexus cyst; metrizamide ventriculography; computed tomography. mission, her headache and visual symptoms had improved spontaneously.

On admission to the University of Michigan Hospital on February 29, 1980, she was noted to be obese. Examination showed normal mental status. Positive neurological findings included only 4+ papilledema bilaterally and loss of spontaneous venous pulsations. The vision was $20 / 20$ bilaterally with full visual fields, and the remainder of her general neurological examination was negative.

The patient was evaluated initially with a contrast enchanced CT scan (Fig. 1), which showed a large lowdensity area in the region of the right lateral ventricle with a mild shift of the midline structures to the left, and an anterior shift of the right choroid plexus. The density of the lesion was that of cerebrospinal fluid, and it was considered to be either a mass within the ventricle or a localized enlargement of the right lateral ventricle. Carotid and vertebral angiography showed only an avascular area with mass effect in the same region. Plain roentgenograms of the skull and chest were normal.

At that point, a definitive diagnosis could not be made, and the decision was made to proceed with metrizamide ventriculography coupled with $C T$ scanning in an attempt to delineate further the nature of the lesion. A right parietooccipital burr hole and ventriculostomy were made to allow the administration of metrizamide into the ventricle without the risk of lumbar puncture. Ventriculography (Figs. 2, 3) and CT scanning (Fig. 4) showed the presence of a $7-\mathrm{cm}$ cyst within the antrum of the right lateral ventricle. Dye had been introduced both within the cyst and within the ventricle demonstrating a high-grade obstruction of the right lateral ventricle posterior to the foramen of Monro, with resultant dilatation of the occipital horn.

The patient was taken to the operating room, and the lateral ventricle was entered via a right parietotemporal cortical incision; a large thin-walled cyst was easily identified. Using magnified vision, the cyst was separated from the ependymal walls and found to originate from the choroid plexus. The cyst was punctured and collapsed, and it was removed with the choroid plexus as one mass. The cyst contained crystal-clear fluid under pressure.

Histological examination of the neoplasm (Fig. 5) 

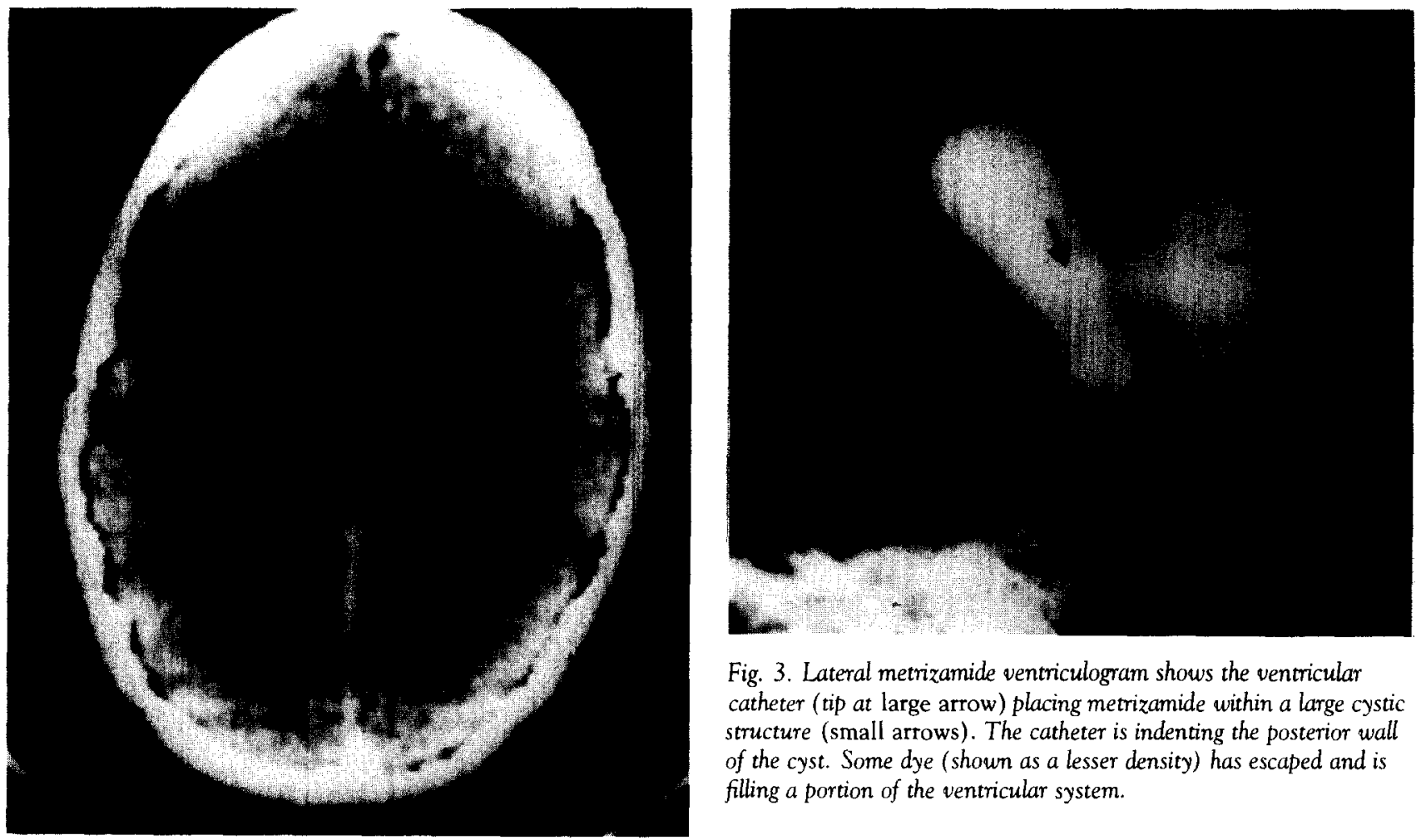

Fig. 3. Lateral metrizamide ventriculogram shows the ventricular catheter (tip at large arrow) placing metrizamide within a large cystic structure (small arrows). The catheter is indenting the posterior wall of the cyst. Some dye (shown as a lesser density) has escaped and is filling a portion of the ventricular system.

Fig. 1. Computerized tomogram (CT) demonstrates low-density area in the region of the posterior right lateral ventricle with shift of midline and lateral displacement of enhancing structure considered to be the right choroid plexus (arrow).

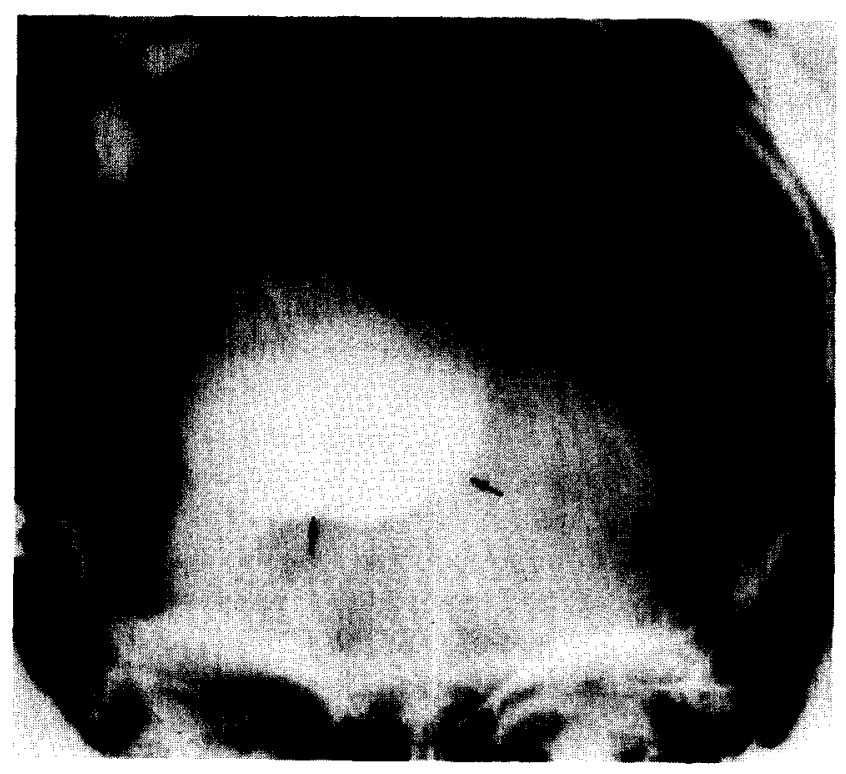

Fig. 2. Anteroposterior film of the metrizamide in a large cystic structure (arrows) located in the region of the right lateral ventricle and crossing the midline.

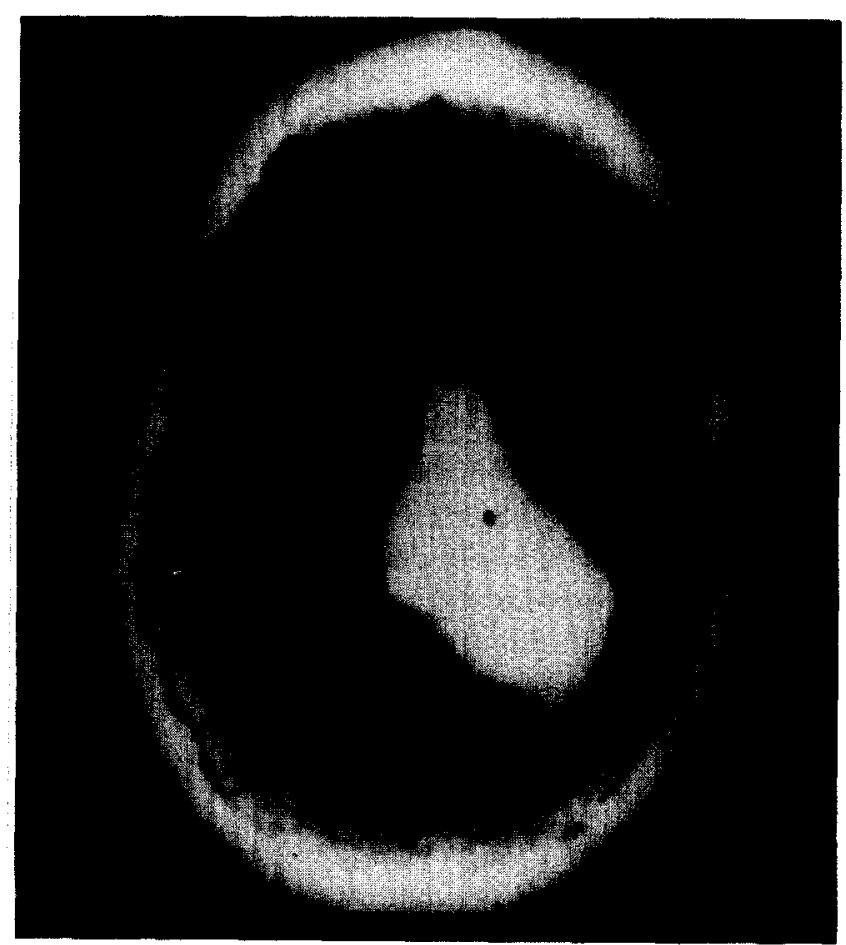

Fig. 4. CT after intraventricular placement of metrizamide shows high-density area outlining a huge cystic mass filling the posterior portion of the right lateral ventricle. Some dye has escaped and outlines a portion of the ventricular system at lower density. 


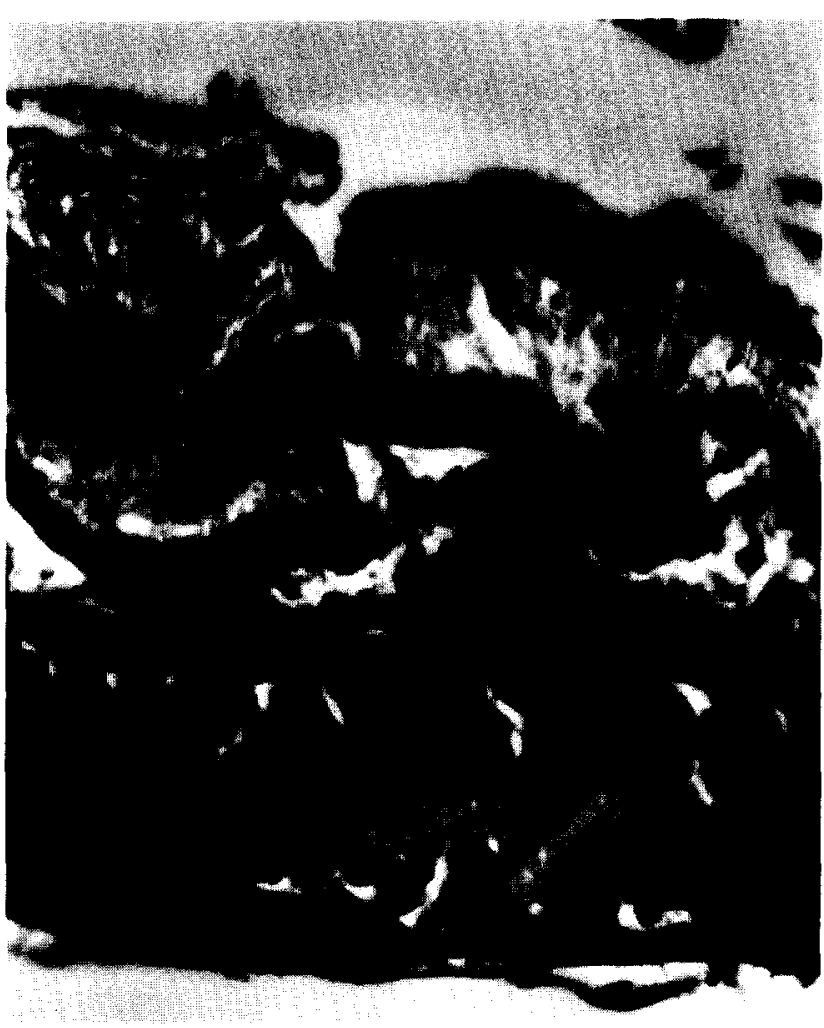

Fig. 5. Photomicrograph of specimen of cyst wall shows thin fibrous connective tissue membrane with a thin lining of columnar epithelium.

showed a large thin-walled cystic cavity originating from otherwise normal choroid plexus. The cyst wall was a thin fibrous membrane lined with low columnar epithelium. The wall contained no evidence of glial elements or malignant changes.

Postoperatively the patient was noted to have extinction to double simultaneous stimulation in both the left arm and the left visual field. Both of these cleared completely over the next three days. Her postoperative course was otherwise uneventful. At discharge she continued to have $20 / 20$ vision bilaterally with full visual fields, and she was free of headache. One month postoperatively her papilledema had resolved and she was symptom free.

\section{Discussion}

Symptomatic cysts of the choroid plexus of the lateral ventricle are believed to originate with the development of the choroid plexus. The choroid plexus of the lateral ventricle is formed by invagination of vascular mesenchyme. The cysts are believed to represent neuroepithelial folds that may fill with cerebrospinal fluid or cellular debris [13]. Unlike other cysts, such as that described by Yeates and Enzmann [16], these contain no glial elements or evidence of malignant origin.

This lesion is believed to have been first described by
Brown [4]. In 1876 he investigated a 4-year-old who died with clinical evidence of acute intracranial hypertension. Brown identified a cyst but it was not confirmed histologically. Five proved cases of cysts 1.6 to $8 \mathrm{~cm}$ in diameter in children 4 months to 10 years of age have been reported [ 1 , $2,6,7,9]$. These cases were characterized by symptoms of increased intracranial pressure $[1,4,7,9]$ often with episodic worsening $[1,2,9]$, suggesting intermittent obstruction of the ventricular system. Normal levels of protein in the cerebrospinal fluid were found in all patients so examined $[2,6,7,9]$.

This case is the first in which this condition has been found in an adult; it appears that although the cyst is probably congenital in origin, it need not reach a size to cause ventricular obstruction until adulthood. Since these cysts generally are attached to the choroid plexus at only one point [10], they may float freely within the ventricle and intermittently obstruct the circulation of ventricular fluid, giving the clinical picture of episodic worsening. They may also enlarge to the point of total obstruction. Presence of symptoms then depends on both the size and the exact location of the cyst within the ventricle.

The diagnosis of this lesion has been greatly aided by the advent of CT scanning. However, contrary to the opinion of Giorgi [7], even this technique has not been sufficient to provide precise preoperative diagnosis. This inadequacy was predicted by de la Torre [5]. The enhanced CT scan shows location, but not the nature of the lesion. For this reason, we feel that the combination of metrizamide introduced by ventriculostomy and CT scanning gives the maximum information. The use and relative safety of this water-soluble contrast agent for ventriculography has been established over the past eight years in several international studies [3, 8]. Though the spinal route of introducing metrizamide shows promise in investigation of intracranial abnormalities such as pituitary tumors $[11,12]$, we do not feel that it can be safely utilized in these cases of masses in the lateral ventricle with papilledema. As demonstrated by this case, a reasonable preoperative diagnosis can be made. Although it may not always be possible to place contrast both within the cyst and within the ventricle, the cyst should generally be outlined by the dye as a negative image. The surgeon can then proceed with the expectation that this is a benign lesion that it is possible to completely remove.

The operative approach to this lesion needs to be adapted to the individual patient. It has been suggested by de la Torre [6] that the lesion be approached by a horizontal incision in the midtemporal gyrus. In this case we utilized a posterior parietotemporal incision in the nondominant hemisphere, since this was the thinnest area of cortex over the cyst. Although no permanent deficit resulted, a transient decrease in the ability to recognize double simultaneous stimuli was noted. For these lesions we would also consider the transcallosal approach to the ventricles, especially 
for cases involving a cyst within the lateral ventricle of the dominant hemisphere. In addition to avoiding traversing the dominant parietal lobe, this would tend to allow initial access to the more anterior portion of the cyst where a vascular pedicle may be encountered $[14,15]$.

In summary, a rare case of symptomatic choroid plexus cyst of the lateral ventricle has been reported. This is the first such case described in an adult. Its presentation was typical with episodic increased intracranial pressure, papilledema, and visual changes. Preoperative diagnosis using Metrizamide-enhanced CT scanning and ventriculography was found to be possible.

\section{References}

1. Andreussi L, Cama A, Cozzutto C, Gianbartalomei G, Grossi G: Cyst of the choroid plexus of the left lateral ventricle. Surg Neurol 12:53-57, 1979

2. Baker $G$, Gottlieb $C$ : Cyst of choroid plexus of the lateral ventricle: Case report. Mayo Clin Proc 31:95-97, 1956

3. Bloch B, Ginacchio A: Metrizamide: A non-ionic water soluble contrast medium-experimental and preliminary clinical investigation. Acta Radiol [Suppl] (Stockh) 335:14-24, 1973

4. Brown G: Cyst of choroid plexus of large size in an infant. Trans Pathol Soc London 27:25-26, 1876
5. De la Torre E: Comment. Neurosurgery 5:56, 1979

6. De la Torre E, Alexander E, Davis C, Crandell L: Tumors of the lateral ventricle of brain: report of eight cases, with suggestions for clinical management. J Neurosurg 20:461-470, 1963

7. Giorgi C: Symptomatic cyst of the choroid plexus of lateral ventricle. Neurosurgery 5:53-56, 1979

8. Gansette R: Metrizamide as a contrast medium for myelography and ventriculography. Acta Radiol [Suppl] (Stockh)335:346-358, 1973

9. Neblett C, Robertson J: Symptomatic cysts of the telencephalic choroid plexus. J Neurol Neurosurg Psychiatry 34:324-328, 1971

10. Netsky M, Shuangshoti S: The choroid plexus in health and disease, Charlottesville: University Press of Virginia, 1975, pp 351

11. Robertson G, Brismar J, Weiss A, Davis K, Taveras J, New F, Ackerman $R$, Glenn W: CSF enhancement for computerized tomography. Surg Neurol 6:235-238, 1976

12. Robertson G, Kleefield J, Ellis G, Taveras J, Davis K: Metrizamide enhancement of cerebrospinal fluid for computer tomography. Acta Radiol [Suppl] (Stockh)335:306-313, 1977

13. Shuangshoti S, Roberts M, Netsky M: Neuroepithelial (colloid) cysts. Pathogenesis and relation to choroid plexus and ependyma. Arch Pathol 80:214-224, 1965

14. Stein B: Transcallosal approach to third ventricular tumors, in Schmidek H, Sweet W (eds): Current Techniques in Operative Neurosurgery. New York: Grune \& Stratton, 1977, pp 247-255

15. Taren J: Tumors at the diencephalon-transcallosal approach to the third ventricle, in Kahn E, Crosby E, Schneider R, Taren J: Correlative Neurosurgery, ed 2. Springfield, Ill: Thomas, 1969, p 139

16. Yeates A, Enzmann D: Case report: an intraventricular arachnoid cyst. J Comput Assist Tomogr 3:697-700, 1979 